\title{
High-resolution Multimodal Confocal Raman-imaging in SEM by Means of RISE Microscopy
}

Jan Englert, Ute Schmidt, Olaf Hollricher and Harald Fischer

WITec Wissenschaftliche Instrumente und Technologie GmbH, Ulm, Baden-Wurttemberg, Germany

The characterization of materials, may it be hard or soft, organic or inorganic matter, often requires more than a single analytical technique to gather comprehensive information. Especially fully understanding and subsequently predicting the behavior for specific applications can be a challenging task. The scope of this presentation is to introduce a tool which blends in seamlessly in existing high-resolution SEM imaging capabilities while providing chemically highly specific 3D information at the limit of optical diffraction. Furthermore, we wish to present how a combination of spectroscopic methods (e.g. Raman/Photoluminescence) and microscopy techniques (confocal/AFM/SEM) can contribute to your in depth understanding of above-mentioned materials.

Linking of data originating various methods conducted at the same sample position does always deliver a more comprehensive insight into the material, however having those methods available in a common platform accelerates the workflow, increases the level of reliability and simplifies interpretation. We wish to present our multimodal microscopy approach to make 3D confocal Raman imaging accessible on nanoanalytical platforms including but not limited to SEM. In a series of examples, we will demonstrate how this approach allows for the characterization of phase separations in polymer blends, facilitates understanding of complex geological samples or provides in depth information in lithium battery research.

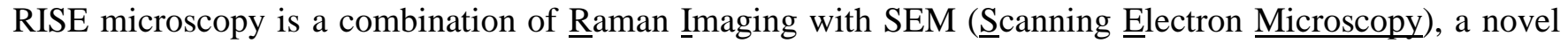
correlative microscopy technique, which combines the Raman effect with high spatial resolution scanning electron microscopy within one single integrated microscope system. SEM typically is equipped with various accessories and modes of detection (SE - secondary electrons, BSE - backscattered electrons) and especially along with microanalysis tools (EDS, CL) can be a particularly powerful tool for research, development, quality control or failure analysis. SEM on the one hand can provide information on morphology, elemental composition and crystallography. 3D Confocal Raman imaging on the other hand will provide in depth analysis of polymorphs beyond elemental composition and vivid details on soft matter (e.g. polymers or organic matter) corroborated by information on orientation, crystallinity, strain and anisotropies.
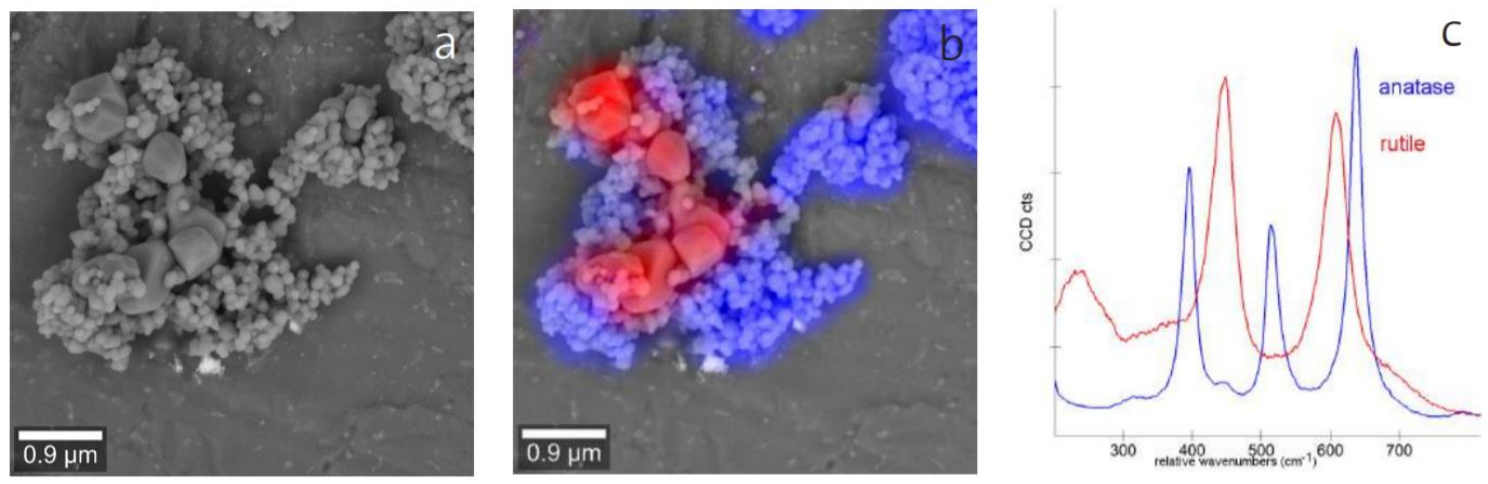

Figure 1. Two modifications of $\mathrm{TiO} 2$, anatase and rutile, were mixed 1.1, ground, dissolved in water and imaged with an SEM (a) and a confocal Raman microscope. (b) SEM and Raman images overlaid. (c) In the Raman spectrum anatase (blue) can be easily distinguished from rutile (red). Image parameters: 12 x $12 \mu \mathrm{m} 2$ scan range, $150 \times 150$ pixels $=22,500$ spectra, integration time: $0.037 \mathrm{~s} /$ spectrum 

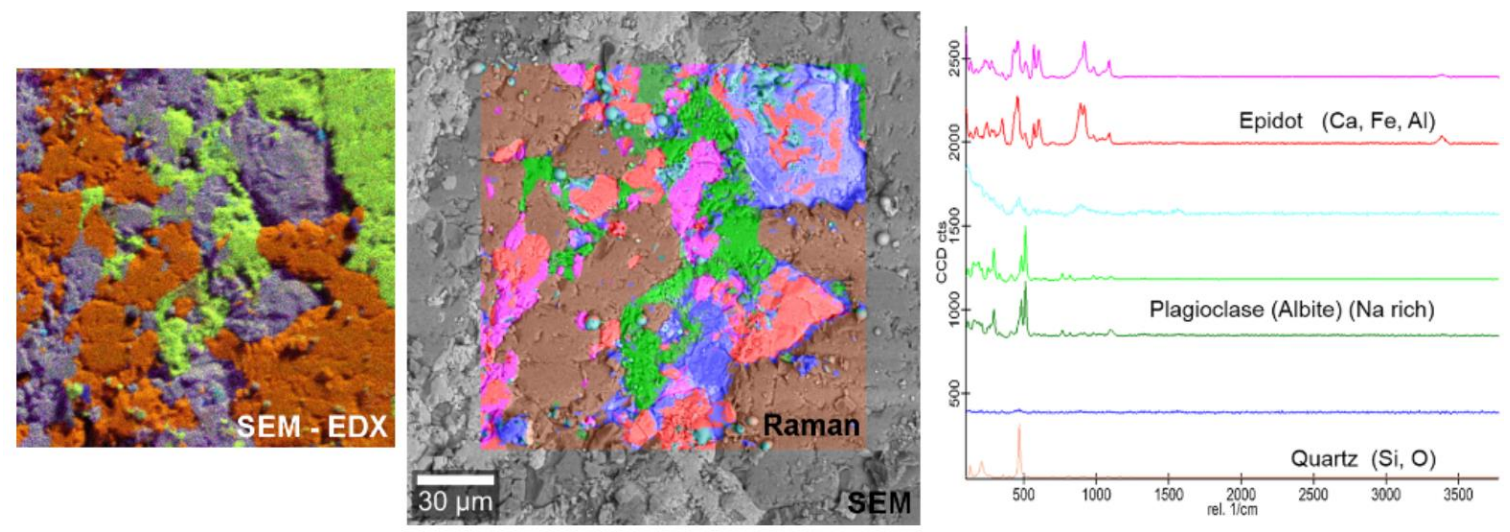

Figure 2. RISE Microscopy and EDX analysis of a geological sample. Left: Overlaid SEM-EDX image: Three different element groups can be distinguished (Orange: Si, O; Purple: Si, Al, Fe, Ca; Green: Na). Middle: The Raman-SEM image overlay of the same sample area shows the distribution of the molecular compounds. Right: Corresponding Raman spectra. Red: Epidote; Green: Quartz; Brown: Plagioclase (Albite); Plus additional molecular compounds.

\section{References}

Schmidt, R.; Fitzek, H.; Nachtnebel, M.; Mayrhofer, C.; Schroettner, H.; Zankel, A., The Combination of Electron Microscopy, Raman Microscopy and Energy Dispersive X-Ray Spectroscopy for the Investigation of Polymeric Materials. Macromolecular Symposia 2019, 384 (1), 1800237

Wille, G.; Lerouge, C.; Schmidt, U., A multimodal microcharacterisation of trace-element zonation and crystallographic orientation in natural cassiterite by combining cathodoluminescence, EBSD, EPMA and contribution of confocal Raman-in-SEM imaging. Journal of Microscopy 2018, 270, 309-317.

Schmidt, U.; Hollricher, K.; Ayasse, P.; Hollricher, O., Correlative RISE microscopy: Raman imaging meets scanning electron probe microscopy. Microscopy and Analysis 2015, 29 (3), 24-27.

Stolcova, L.; Prosika, J.; Vana, R.; Dluhos, J., Correlative RISE microscopy and 3D reconstruction. Imaging \& Microscopy 2015, 17.

Schmidt, U.; Liu, W.; Yang, J.; Dieing, T.; Weishaupt, K., The power of confocal raman-AFM and ramanSEM (RISE) imaging in polymer research. In Microscopy and Microanalysis, 2015; Vol. 21, pp 2189-2190. 